\title{
International Seminars in Surgical

\section{Preoperative bi-fractionated accelerated radiation therapy for combined treatment of locally advanced rectal cancer in a consectutive series of unselected patients} Roberto Biffi*†1, Hugo Marsiglia ${ }^{\dagger 2,3}$, Barbara Jereczek Fossa ${ }^{\dagger 2}$, Maria Cristina Leonardi ${ }^{\dagger 2}$, Domenico Cante ${ }^{\dagger 2}$, Roberta Lazzari ${ }^{\dagger 2}$, Antonio Chiappa ${ }^{\dagger 1}$, Sabine Cenciarelli ${ }^{\dagger 1}$, Bruno Andreoni ${ }^{\dagger 1}$, Maria Giulia Zampino $^{\dagger 4}$ and Roberto Orecchia ${ }^{\dagger 2}$

\footnotetext{
Address: ${ }^{1}$ Dept. of General Surgery-European Institute of Oncology and University of Milano, Via Ripamonti, 435 , Italy, ${ }^{2}$ Dept. of RadiotherapyEuropean Institute of Oncology and University of Milano, Via Ripamonti, 435, Italy, ${ }^{3}$ Dept. of Radiotherapy, Institute Gustav Roussy, Villejuif, France and ${ }^{4}$ Dept. Of Oncolgy-European Institute of Oncology-Milano, Via Ripamonti, 435. Italy

Email: Roberto Biffi* - roberto.biffi@ieo.it; Hugo Marsiglia - roberto.biffi@ieo.it; Barbara Jereczek Fossa - barbara.fossa@ieo.it; Maria Cristina Leonardi - mariacristina.leonardi@ieo.it; Domenico Cante - roberto.biffi@ieo.it; Roberta Lazzari - roberta.lazzari@ieo.it; Antonio Chiappa - antonio.chiappa@unimi.it; Sabine Cenciarelli - sabine.cenciarelli@ieo.it; Bruno Andreoni - bruno.andreoni@ieo.it; Maria Giulia Zampino - mariagiuliazampino@ieo.it; Roberto Orecchia - roberto.orecchia@ieo.it

* Corresponding author †Equal contributors
}

Published: 20 September 2007

International Seminars in Surgical Oncology 2007, 4:23 doi:10.1 I86/1477-7800-4-23

This article is available from: http://www.issoonline.com/content/4/I/23

2007 Biffi et al; licensee BioMed Central Ltd.

This is an Open Access article distributed under the terms of the Creative Commons Attribution License (http://creativecommons.org/licenses/by/2.0), which permits unrestricted use, distribution, and reproduction in any medium, provided the original work is properly cited.

\begin{abstract}
Background: although preoperative RT (Radiation Therapy) is becoming the preferred approach for combined treatment of locally advanced rectal adenocarcinoma, no regimen can be now considered as a standard. Since the toxicity of preoperative RT isn't yet completely known, and the advantages of preoperative RT could be counterbalanced by increased postoperative morbidity and mortality, a monocentre series of preoperative bifractionated accelerated RT was retrospectively reviewed to clarify toxicity and outcomes after a prolonged follow up.

Methods: patients were screened following these eligibility criteria: histology-proven adenocarcinoma of the rectum; distal tumour extent at $12 \mathrm{~cm}$ or less from the anal verge; clinical stage T3-4/anyN, or any T/NI-2; ECOG Performance Status 0-2. A total dose of 4I.6 Gy (26 twice daily fractions of $\mathrm{I} .6 \mathrm{~Gy}$ ) was delivered. Surgery was carried out $17 \pm 2$ days after RT completion, adopting the total mesorectal excision technique.

Results: 24 men and 23 women were enrolled; median age was 55 years ( $r .: 39-77)$. Twenty-eight patients were stage II and 19 stage III. 9 patients suffered from a recurrent tumour. 2 patients experienced a severe grade 4 gastrointestinal toxicity (a colo-vaginal fistula and an intestinal obstruction, both successfully treated). Operative mortality was nil; postoperative early complications occurred in 13 cases; mean length of hospital stay was 15 days. After a mean follow up of 44 months ( $r .:$ 18-84) 8 patients had deceased for recurrent disease, 15 were alive with a disease progression ( 2 pelvic recurrences and 13 pure distant deposits) and 24 were alive, without disease. The 5 -year actuarial overall survival was $74.2 \%$, the disease-free survival $62.9 \%$ and the regional control rate $84.7 \%$. Long-term complications included I case of radiation enteritis requiring surgery, 2 cases of anastomotic stricture and 3 cases of bladder incontinence.
\end{abstract}




\begin{abstract}
Conclusion: bifractionated accelerated RT administered in the preoperative setting to patients bearing locally advanced rectal cancer is reliable and safe, as its immediate and late toxicity (mainly infectious) is acceptably low and long-term survivals are achievable. These findings support the increasing use of preoperative RT for treatment of this malignancy in experienced centres. Ongoing multicentric trials are expected to address still unsolved issues, including the benefit of CT adjunct to preoperative RT.
\end{abstract}

\section{Background}

Surgery remains the primary modality of treatment for rectal cancer and is the only therapy required for earlystage disease. Locally advanced rectal adenocarcinoma (i.e. resectable cancer with transmural spread and/or lymphatic nodes involvement) continues indeed to represent a major challenge to surgeons. Although remarkable progress has been made during the past two decades in improving surgical techniques, tumours in the lower half of the rectum pose specific technical problems, and a radical R0 resection of the tumour and its lymphatic component may be difficult to obtain, especially when a huge lesion is located in a very narrow pelvis of a male patient. Several studies have recently focused attention on the importance of standardized surgical technique of total mesorectal excision $[1,2]$ as well as its integration with chemo-(CT) and radiation therapy (RT) for optimal treatment of this malignancy $[3,4]$. Although preoperative RT is becoming the preferred approach to locally advanced rectal cancer in many Institutions and large randomised trials have shown that preoperative RT can substantially decrease local failure rates and slightly improve overall survival $[5,6]$, no RT regimen can be now considered as a standard, and ongoing studies are still addressing some major unresolved issues, including the optimal timing of RT (preop- vs postoperative) and the relative merits of short vs long-course RT. A major concern raised from a recent meta-analysis, showing a consistent increase in noncancer-related mortality for patients receiving preoperative RT [7]; since the toxicity of preoperative RT seems to be not yet completely known, and the advantages of preoperative RT could be counterbalanced by increased postoperative morbidity and mortality, as seen in some trials [8], we decided to retrospectively review our series of preoperative bi-fractionated accelerated RT in locally advanced rectal cancer, in the attempt to identify risk factors for early and late toxicity (if any), and to define most significant oncologic and functional outcomes after a prolonged follow up.

\section{Methods}

After being informed and giving their consent, 47 consecutive patients from the European Institute of Oncology in Milan were enrolled in this study. The eligibility criteria included the following: histology-proven adenocarcinoma of the rectum; distal tumour extent within $12 \mathrm{~cm}$ from the anal verge; clinical stage $\mathrm{T} 3-4 / \mathrm{anyN}$, or anyT/ N1-2 for primary or recurrent disease as well; no evidence of distant deposits; ECOG Performance Status 0-2; age $>18$ years; no prior or concurrent malignancy, and finally no prior RT. Adjuvant CT was allowed for eligible patients, suffering from recurrent carcinoma of the rectum.

Pre-treatment evaluation included complete physical examination, digital rectal examination, common laboratory biochemical test, CEA (Carcinoembryonic Antigen) determination, colonoscopy, CT-scan (Computerized Tomography) of the thorax, abdomen and pelvis, and EUS (endoscopic rectal ultrasonography). Total dose delivered was $41.6 \mathrm{~Gy}$ in 26 fractions of $1.6 \mathrm{~Gy}$, two times daily with at least a 6 -h interval. The target volume included the tumour and each enlarged lymph node (if any), with a margin of at least $3 \mathrm{~cm}$, perirectal and internal iliac lymph nodes, the presacral area and surrounding organs considered to be involved by tumour. A four-field technique (box) was always used. Dosimetry was optimised by means of a treatment-planning computerized program, on the basis of either patient's contours or dosimetric CT scan. High energy photons of at least $15 \mathrm{MV}$ were used.

Surgery was scheduled after a rest period of $17 \pm 2$ days from RT completion. Total mesorectal excision technique was always adopted. Adjuvant CT (5 FU + Folinic Acid bolus) for a 6-month period was planned for patients who had $\mathrm{T} 4$ or $\mathrm{N}+$ at pathology report of surgical specimen.

\section{Results}

Table 1 summarizes patients' population pertinent characteristics. 47 patients were treated (24 men and 23 women); the median age was 55 years (r.: 39-77). Pretreatment clinical staging included 28 cases stage II and 19 stage III. 9 patients suffered from a recurrent tumour. Median distance between the lower tumour edge and the anal verge was $5 \mathrm{~cm}$ (range: $<1-12$ ).

Three patients did not complete the planned RT schedule as a consequence of early toxicity; in these cases the dose administered was 32, 40 and 40.8 Gy respectively.

Table 2 shows the observed toxicity; severe grade 4 gastrointestinal reactions included a colo-vaginal fistula, 
Table I: patients' population pertinent characteristics

\begin{tabular}{ll}
\hline No. of patients treated & 47 \\
Male: Female ratio & $24: 23$ \\
Mean age (range) & $55 \mathrm{yrs} .(39-77)$ \\
Primary tumours : recurrences ratio & $38: 9$ \\
cTNM Stage II : stage III ratio & $28: 19$ \\
$\begin{array}{l}\text { Mean distance between tumour edge and anal } \\
\text { verge (range) }\end{array}$ & $5.0 \mathrm{~cm}(<1-12)$ \\
$\begin{array}{l}\text { No. of patients who completed RT programme } \\
\text { as protocol }\end{array}$ & $44(93.6 \%)$ \\
\hline
\end{tabular}

while 1 patient suffered from grade 3 toxicity (intestinal sub-obstruction). Both were successfully treated. 9 cases of grade 2 and 19 cases of grade 1 gastrointestinal reactions were detected. One case of grade 2 and 15 cases of grade 1 urogenital morbidity were observed. 6 cases of grade 2 and 6 cases of grade 1 haematological toxicity occurred. Finally, one grade 2 and 7 grade 1 skin toxicity were observed.

Surgery was performed at a mean interval of $17 \pm 2$ days after RT completion, always adopting the total mesorectal excision technique. Table 3 lists the main surgical features; abdomino-perineal resection (APR) was carried out in 14 patients, and low anterior resection with mechanical anastomosis in 33.16 patients had blood peroperative transfusions; a mean of 2 units of packed red blood cells were administered ( $\mathrm{r} .=0-5)$. All operated patients could benefit of a curative surgery, expressed by an R0 procedure (proximal, distal and circumferential margins free of disease; the circumferential free margin resulted less than 1 $\mathrm{mm}$ in 9 cases).

None patient obtained a complete pathology response to the treatment. Downstaging, expressed as pathologic ypT1-2, ypN0 stage, occurred in 3 patients only. A postRT pathology stage ypT1 was detected in 1 case, ypT2 in 7, ypT3 in 33 and ypT4 in 6 . Positive nodes were detected in 33 patients. Mean length of hospital stay was 15 days (range: 8-35). YpT4-any $\mathrm{N}$ and $\mathrm{N}$ positive patients under-

Table 2: observed acute toxicity from preoperative radiation therapy

\begin{tabular}{lcccc}
\hline Type & Grade I & Grade 2 & Grade 3 & Grade 4 \\
\hline Gastrointestinal & 19 & 9 & $\begin{array}{c}\text { I (intestinal } \\
\text { obstruction) }\end{array}$ & $\begin{array}{c}\text { I } \\
\text { (colovaginal } \\
\text { fistula)** }\end{array}$ \\
Urogenital & 15 & 1 & - & - \\
Haematologic & 6 & 6 & - & - \\
Skin & 7 & 1 & - & - \\
\hline
\end{tabular}

* successful conservative treatment; ** successful en-bloc resection at the time of programmed surgery
Table 3: main surgical and pathology features in 47 operated patients

\begin{tabular}{lcc}
\hline Parameter & No. & $\%$ \\
\hline Low anterior resection & 33 & 70.2 \\
Abdomino-perineal resection & 14 & 29.8 \\
En-bloc resection of other organs & 5 & 10.6 \\
R-0 operation Circumferential free margin < I & 479 & 10019.1 \\
mm & 9 & 19.1 \\
Circumferential free margin < I mm & 9 & 19.1 \\
Patients having blood peroperative transfusions & & \\
(mean: 2 PRBC; r: 0-5) & 3 & 6.3 \\
Downstaging (yPTI-2 ypN0) & 8 & 17.0 \\
ypTI - yPT2 & 39 & 83.0 \\
ypT3 - yPT4 & 15 & \\
Mean length of hospital stay (days; r) & $(8-$ & \\
& $35)$ & \\
& & \\
\end{tabular}

went adjuvant chemotherapy for a median of 6 months ( 5 FU + Folinic Acid bolus).

Postoperative complications occurred in 13 cases (a detailed list is presented in Table 4): two anastomotic leaks (both requiring a re-operation), two cases of prolonged postoperative ileus, one pelvic abscess (causative agent: $P$. aeruginosa) and eight cases of surgical site infection (SSI) grade I-II, according to CDC classification (perineal and/or laparotomy wound).

After a mean follow up of 44 months (range: 18-84) (Table 5), six patients experienced severe late complications: three cases of grade 3 gastrointestinal toxicity ( 1 radiation enteritis requiring surgery and 2 cases of anastomotic stricture requiring serial endoscopic dilatations) and three cases of grade 3 urogenital toxicity (vescical incontinence). No case of severe cardiovascular toxicity occurred. 8 patients ( 6 of them affected from recurrence of previous rectal cancer) had deceased for recurrent disease (pelvic recurrence and concomitant distant metastases), 15 were alive with a disease progression ( 2 pelvic recurrences and 13 pure distant deposits) and 24 were alive, without disease. The 5 year actuarial overall survival was $74.2 \%$, the disease-free survival $62.9 \%$ and the regional control rate $84.7 \%$.

Table 4: postoperative early complications

\begin{tabular}{lcc}
\hline Type & No. of cases & $\%$ \\
\hline Anastomotic leak & 2 (out of 33) & 6.0 \\
Prolonged postoperative ileus & 2 (out of 47) & 4.2 \\
Abdominal abscess & I (out of 47) & 2.1 \\
Surgical site infections (grade I-II)* & 8 (out of 47) & 17.0 \\
All & I3 (out of 47) & 27.6 \\
\hline
\end{tabular}

*according to CDC classification of Surgical Site Infections 
Table 5: long term results (mean follow up: 44 mo., r. = 18-84)

\begin{tabular}{lcc}
\hline Parameter & No. & $\%$ \\
\hline $\begin{array}{l}\text { Deceased } \\
\text { (progression of } \\
\text { disease) }\end{array}$ & 8 & 17.0 \\
$\begin{array}{l}\text { Primary tumour } \\
\text { Recurrent disease }\end{array}$ & 2 & 25 \\
$\begin{array}{l}\text { Alive without } \\
\text { disease }\end{array}$ & 6 & 75 \\
$\begin{array}{l}\text { Alive with disease } \\
\text { Overall local } \\
\text { recurrences }\end{array}$ & 15 & 51.0 \\
$\begin{array}{l}\text { Pure distant } \\
\text { metastases }\end{array}$ & 10 & 31.9 \\
$\begin{array}{l}\text { Urogenital toxicity } \\
\text { grade 3 } \\
\text { Gastrointestinal } \\
\text { toxicity - grade } 3\end{array}$ & $13 *$ & 21.2 \\
\hline
\end{tabular}

\footnotetext{
* liver 7 cases, lung 4 cases, peritoneum and lymphnodes 2 cases

** bladder incontinence

*** I case of radiation enteritis requiring surgery, 2 cases of

anastomotic stricture requiring serial endoscopic dilatations
}

\section{Discussion}

At present, a variety of innovative RT schedules aiming at obtaining minimal treatment toxicity are being tested, including preoperative bi-fractionated accelerated RT. Theoretic common advantages of long- and short-course of preoperative RT include the better tumour radio sensitivity, coming from improved oxygenation of presurgical field, the reduction in tumour seeding by surgical handling and perhaps the reduced toxicity, due to the lesser amount of radiated small bowel. A recent meta-analysis of studies dealing with adjuvant RT for rectal cancer, including over 8,000 patients, has reported a reduction of local recurrence risk by almost $50 \%$ by preoperative treatment, independently from fractionation schedules, compared to $37 \%$ of risk reduction for postoperative RT [7]. Practical and economic considerations provided a major effort for developing short-course treatments, as the tumoricidal effect of short-course RT (25 Gy in one week) seems to be equal to that of long-course (42 to 50 Gy over four to six weeks), using a variety of schedules [9-11]. Unfortunately, a single randomised study has compared preoperative short-course with postoperative RT, delivered over 8 weeks [12]; the reduced local recurrence rate with the former does not mean a inherent superiority of short vs long-course and adds little to the debate regarding this issue. However, a major concern could be the price for short-course preoperative RT, in terms of increased shortand long-term toxicity, as previously observed with shortcourse RT at other sites. This concern has been highlighted in the above mentioned meta-analysis, which showed a significant increase in noncancer-related mortality at one year of follow up, mainly as a consequence of cardiovascular events in older patients [7]. We did not observe sim- ilar events in our experience, and is likely that could be the result of outdated techniques, as there was no increase in postoperative mortality in patients receiving preoperative RT according to modern techniques in either the Swedish Rectal Cancer Trial or the Dutch Trial. With respect to early toxicity, the investigators from the Swedish trial reported an increased frequency of postoperative fistulas and femoral neck and pelvic fractures; moreover, for patients who underwent a conservative surgery, a significantly altered residual sphincter function was found [5]. The Dutch group reported an increase in perineal complications (essentially infectious) in patients receiving preoperative RT compared to surgery alone controls [6]. Our own data confirm these findings: the vast majority of early observed toxicity is related to infectious complications of surgical wounds, whereas anastomotic leaks and pelvic abscess are less relevant.

Our single case of late small bowel toxicity confirms another previous evidence, coming from randomised trials and retrospective experiences, that preop-RT may have fewer adverse effects on long-term bowel function than postoperative therapy $[13,14]$. One possible explanation could be the fact that the radiated rectosgmoid is removed after neoadjuvant therapy; moreover, this could be the result of a lower radiation dosage to the small bowel, as it is much easier to exclude the small intestine from the radiation field in a pelvis free of surgical adhesions by proper positioning of the patients [15]. Moreover, postoperative treatment is associated with increased frequency of bowel movements per day, higher rate of incontinence and increased use of antidiarrhea medications [16]. In addition, a large German multi-institutional study reported that only $37 \%$ of expected patients were referred for postoperative adjuvant treatment, reflecting a surgeons' policy based on patients' individual risk factors evaluation instead of TNM stage [17]. In our non-randomised study, no patients who were continent before treatment developed incontinence, thus comparing favourably with the incontinence rates associated with postoperative adjuvant treatment.

A possible benefit of preoperative therapy is the potential for downstaging, thus increasing resectability rate and the possibility of sphincter-preserving surgery. Although the extent of downstaging appears related to the time interval between completion of preop RT and surgery, the two major studies of preoperative radiation therapy in rectal cancer (Swedish [5] and Dutch [6] trials) scheduled surgery after 1 week and 10 days respectively after the end of radiation treatment; moreover, in the Swedish trial more patients in the preoperative RT group had Dukes stage A or B cancers, whereas in our series only patients with locally advanced or recurrent disease have been treated. In the larger Dutch study no difference in the rates of positive 
circumferential margins has been detected (16\% in the preoperative RT vs $19 \%$ of surgery alone); our own data confirm this evidence: a short course of preop-RT and a short time-interval before surgery are not able to produce a significant pathologic downstaging, and complete responses should not be expected. Our finding of 9 out 47 patients with minimal circumferential free margin at surgery (less than $1 \mathrm{~mm}$ ) confirms that the biological effect of radiation can extend beyond the anatomic boundaries of the lesion, with minimal damage to surrounding normal tissues.

Minsky et al. studied 22 patients with distal rectal cancers that had been deemed to require APR for cure, and found that preoperative RT resulted in significant tumour downstaging, enabling approximately $90 \%$ of patients to undergo sphincter preservation surgery [18]. Similarly, Rouanet et al. used preoperative irradiation to treat 37 patients with rectal cancer that was thought to require an APR [19]; sphincter salvage was possible in $78 \%$ of patients. Francois and co-workers [20], in the only study specifically designed to explore this issue, randomised a total of 201 patients to either surgery after 2 weeks or after 6-8 weeks from the completion of the RT; both clinical and pathologic downstaging were significantly higher in the longer interval group.

Nonetheless, it's our opinion that achieving a microscopic tumour-free circumferential margin is an important factor affecting local pelvic recurrence, and the surgeon's ability to obtain tumour clearance can be challenged by the physical constraints of a narrow pelvis, particularly in male patients. A short course of preop-RT offers the advantage of decreasing the size ("downsizing") of the tumour before surgery, increasing the resectability rate of lesions which were previously deemed unresectable with curative intent or at high risk of R1-R2 procedures. In our series, patients with recurrent tumours and those with huge masses deeply invading the mesorectum had local recurrence rates that remain very high, in spite of preoperative RT administration; that's why our efforts are now directed to obtain a better local control of these huge lesions by means of combined preoperative CT-RT treatment, using higher doses and longer time interval, in agreement with other groups' reported experiences [21].

\section{Conclusion}

In conclusion, bi-fractionated accelerated RT administered in the preoperative setting to patients suffering from locally advanced rectal cancer was safe in terms of early and long-term toxicity, the latter being expressed as acceptably low postoperative complications' rate (mainly infectious) and toxic effects on small bowel. These findings support the increasing use of preoperative RT in the treatment of locally advanced rectal carcinoma; ongoing trials are expected to address major unsolved issues, including the role of combined modality treatment (RT plus $\mathrm{CT}$ ) in the preoperative setting.

\section{References}

I. Porter GA, Soskolne CL, Yakimetes WW, Newman SC: Surgeonrelated factors and outcome in rectal cancer. Ann Surg 1998, 227: I57-67.

2. Martling AL, Holm T, Rutqvist LE, Moran BJ: Effect of a surgical training programme on outcome of rectal cancer in the County of Stockholm. Stockholm Colorectal Cancer Study Group, Basingstoke Bowel Cancer Research Project. Lancet 2000, 356:93-6.

3. Wolmark N, Wieand HS, Hyams DM, Colangelo L, Dimitrov NV, Romond EH, Wexler H, Prager D, Cruz AB Jr, Gordon PH, Petrelli NJ, Deutsch M, Mamounas E, Wickerham DL, Fisher ER, Rochette H, Fisher B: Randomized trial of postoperative adjuvant chemotherapy with or without radiotherapy for carcinoma of the rectum: National Surgical Adjuvant Breast and Bowel Project Protocol R-02. J Natl Cancer Inst 2000, 92:388-96.

4. O'Connell MJ, Martenson JA, Wieand HS, Krook JE, McDonald JS, Haller DG, Mayer RJ, Gunderson LL, Rich TA: Improving adjuvant therapy for rectal cancer by combining protracted-infusion fluorouracil with radiation therapy after curative surgery. $\mathrm{N}$ Engl J Med 1994, 33 I:502-7.

5. Anonymous: Improved survival with preoperative radiotherapy in resectable rectal cancer. Swedish Rectal Cancer Trial. N Engl J Med 1997, 336:980-7.

6. Kapiteijn E, Marijnen CA, Nagtegal ID, Putter H, Steup WH, Wiggers T, Rutten HJ, Pahlman L, Glimelius B, van Krieken JH, Leer JW, van de Velde C]: Dutch Colorectal Cancer Group. Preoperative radiotherapy combined with total mesorectal excision for resectable rectal cancer. N Engl J Med 200I, 345:638-46.

7. Colorectal Cancer Collaborative Group: Adjuvant radiotherapy for rectal cancer: a systematic review of 8507 patients from 22 randomized trials. Lancet 200I, 358:I29I-304.

8. Holm T, Rutqvist LE, Johansson H, Cedermark B: Postoperative mortality in rectal cancer treated with or without preoperative radiotherapy: Causes and risk factors. $B r J$ Surg 1996, 83:964-8.

9. Kirk J, Gray WM, Watson ER: Cumulative radiation effect. I. Fractionated treatment regimens. Clin Radiol 1971, 22: 145-55.

10. Turesson I, Notter G: The influence of fraction size in radiotherapy on the late normal tissue reaction-I: Comparison of the effects of daily and once-a-week fractionation on human skin. Int J Radiat Oncol Biol Phys 1984, 10:593-8.

II. Fowler JF: The linear-quadratic formula and progress in fractionated radiotherapy. Br J Radiol 1989, 62:679-94.

12. Pahlman L, Glimelius B: Pre- or postoperative radiotherapy in rectal and rectosigmoid carcinoma. Report from a randomized multicenter trial. Ann Surg 1990, 2 I I : I87-95.

13. Paty PB, Enker WE, Cohen AM, Lauwers GY: Long-term functional results of coloanal anastomosis for rectal cancer. $A m J$ Surg 1994, 167:90-5.

14. Birnbaum EH, Dreznik Z, Myerson RJ, Lacey DL, Fry RD, Kodner I, Fleshman JW: Early effect of external beam radiation therapy on the anal sphincter: a study using anal manometry and transrectal ultrasound. Dis Colon Rectum 1992, 35:757-6I.

15. Abdelkarim SA, Bischof S, Nuet P: Impact of the <<Belly Board》 Device on treatment reproducibility in preoperative radiotherapy for rectal cancer. Strahlenther Onkol 2002, I 78:259-62.

16. Kollmorgen CF, Meagher AP, Wolff BG, Pemberton JH, Martenson JA, Illstrup DM: The long term effect of adjuvant postoperative chemoradiotherapy for rectal carcinoma on bowel function. Ann Surg 1994, 220:576-82.

17. Wulf J, Kramer K, van Aaken C, Dietzel F, Lucas D, Pfander K, Schimpke T, Schulze W, Thiel HJ, Zleger K, Flentje M: Outcome of postoperative treatment for rectal cancer UICC stage II and III in day-to-day clinical practice. Results from a retrospective quality control analysis in six institutions in North Bavaria (Germany). Strahlenther Onkol 2004, I 80:5-I4.

18. Minsky BD, Cohen AM, Enker WE, Kelsen DP, Kemeny N, Riechman B, Saltz L, Sigurdson ER, Frankel J: Phase I/II trial of preoperative radiation therapy and coloanal anastomosis in distal invasive 
resectable rectal cancer. Int J Radiat Oncol Biol Phys 1992, 23:387-92

19. Rouanet $P$, Farber JM: Conservative surgery for low rectal carcinoma after high dose radiation. Functional and oncologic results. Ann Surg 1995, 22 1:67-73.

20. Francois Y, Nemoz CJ, Baulieux J, Vignal J, Grandjean JP, Partensky C, Souguet JC, Adeleine P, Gerard JP: Influence of the interval between preoperative radiation therapy and surgery on downstaging and on the rate of sphincter-sparing surgery for rectal cancer: the Lyon R90-0I randomized trial. J Clin Oncol 1999, 17:2396.

21. Rullier E, Goffre B, Bonnel C, Zerbib F, Caudry M, Saric J: Preoperative radiochemotherapy and sphincter-saving resection for T3 carcinomas of the lower third of the rectum. Ann Surg 200I, 234:633-40

Publish with Bio Med Central and every scientist can read your work free of charge

"BioMed Central will be the most significant development for disseminating the results of biomedical research in our lifetime. "

Sir Paul Nurse, Cancer Research UK

Your research papers will be:

- available free of charge to the entire biomedical community

- peer reviewed and published immediately upon acceptance

- cited in PubMed and archived on PubMed Central

- yours - you keep the copyright

Submit your manuscript here:

http://www.biomedcentral.com/info/publishing_adv.asp 